\title{
Adenosine analogue blocks dengue infection
}

\begin{abstract}
Dengue virus (DENV) is a flavivirus transmitted by mosquitoes that affects up to 100 million people each year. Infection can be life-threatening, and there are currently no available vaccines or antiviral therapies. Writing in PNAS, Yin and colleagues describe a novel adenosine analogue that is capable of potently inhibiting DENV infection.

DENV is mainly found in the tropics, and infection is characterized by headache, muscle and joint pains, fever and rash. The majority
\end{abstract}

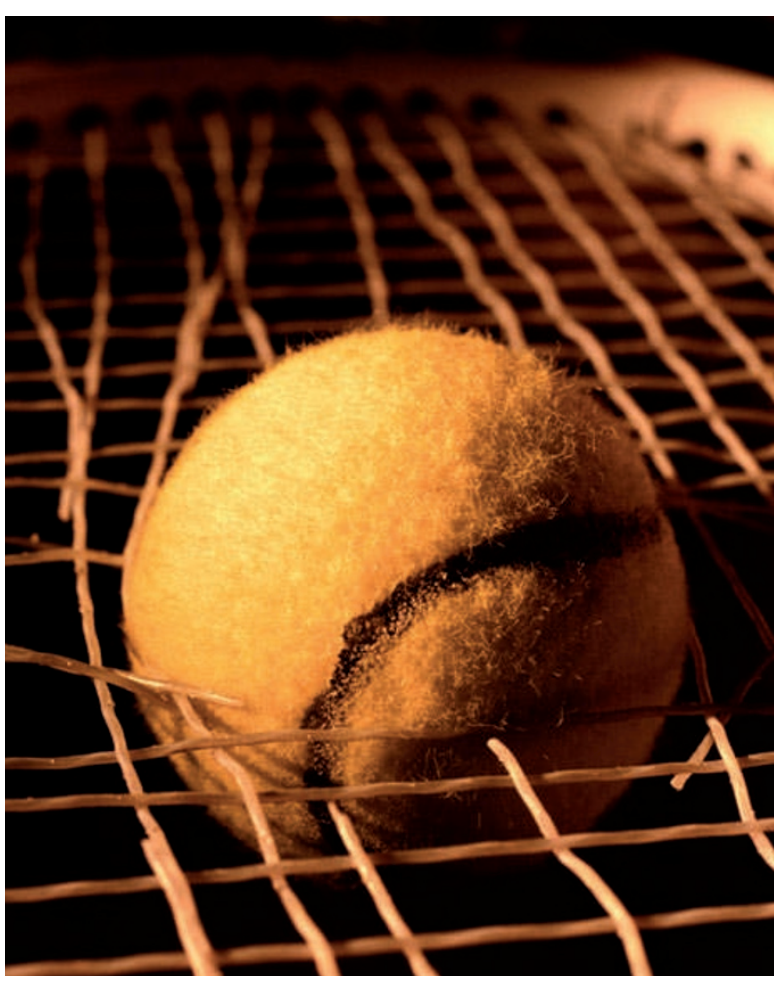

of deaths are due to the development of dengue haemorrhagic fever (DHF) and dengue shock syndrome (DSS). The existence of four DENV serotypes has severely challenged the development of effective treatments. Given that nucleoside analogues have proven effective in combating other viruses, Yin and colleagues set out to determine whether such an approach could similarly be applied to DENV.

First, the authors analysed more than 90 previously undescribed adenosine analogues. NITD008 (which differs from adenosine by a carbon substitution for N-7 of the purine ring and an acetylene group at the 2' position of ribose) was found to be a potent in vitro inhibitor of the DENV RNA-dependent RNA polymerase (RdRp), inhibiting all four DENV serotypes and significantly reducing viral titres in various cell lines. The effects of NITD008 were specific to Flaviviridae family viruses: it similarly inhibited West Nile virus (WNV), yellow fever virus and Powassan virus.

Using a primer extension-based RdRp assay, the authors showed that NITD008 inhibited DENV RdRp by causing termination of RNA chain synthesis. Importantly, continuous culturing of DENV or WNV in cell lines with NITD008 for up to 4 months did not lead to the emergence of resistant strains.

To assess the therapeutic potential of NITD008, mice were infected with DENV-2 and immediately administered NITD008 orally twice daily for 3 days. Peak viraemia and plasma levels of viral non-structural protein 1 (NS1) were suppressed up to 35-fold and 14-fold, respectively. A delayed start of treatment up to 48 hours post-infection also reduced plasma viraemia by $7-10$-fold. Furthermore, similar treatment of a lethal dengue mouse model, in which mice develop hallmark characteristics of DHF and DSS, completely protected mice from death. Mice were also protected when treatment was delayed by up to 24 hours.

Importantly, no observed adverseeffect level could be achieved when rats were dosed orally with NITD008 for 1 week. However, toxicity was observed in rats and dogs treated for 2 weeks. Experiments are in progress to investigate the cause of toxicity and overcome the side effects, although the authors emphasized that treatment duration would be expected to be less than 6 days for this acute disease.

Together, these data support the future development of a nucleoside inhibitor for the treatment of DENV infection. Such an approach might also be broadly effective for the treatment of other flavivirus infections.

Sarah Crunkhorn

ORIGINAL RESEARCH PAPER Yin, Z. et al. An adenosine nucleoside inhibitor of dengue virus. Proc. Natl Acad. Sci. USA 16 Nov 2009 (doi: 10.1073/pnas.0907010106) 